\title{
FREE ENERGIES AND SPECIFIC HEATS OF THE NEMATICS WITHIN NONEXTENSIVE THERMOSTATISTICS
}

\author{
O. Afsar, O. Kayacan \\ Physics Department, Faculty of Sciences and Arts \\ Celal Bayar University, Muradiye, Manisa, Turkey \\ ozhan.kayacan@bayar.edu.tr
}

\begin{abstract}
In this study, the free energies and specific heats of the nematic liquid crystals are studied within Tsallis thermostatistics. In equilibrium, free energy of PAA (para-azoxyanisole) vs the order parameter is plotted, as well as its the specific heat vs temperature. The effect of the nonextensivity is illustrated for some values of the entropic index $q$.
\end{abstract}

Keywords- Tsallis thermostatistics, Maier-Saupe theory, nematic liquid crystals, equilibrium condition.

\section{INTRODUCTION}

The characteristic property of the mesophase of a nematic liquid crystal is its long-range orientational order. The definition of this orientational order is given by

$$
\bar{P}_{2}=\left(\frac{3 \cos ^{2} \theta-1}{2}\right)
$$

which was proposed by Zwetkoff [1], where $\theta$ is the angle between the long axis of the molecule and the director of the mesophase, $\bar{P}_{2}$ is the second Legendre polynomials. The orientational order decreases with the temperature and vanishes suddenly at the nematic-isotropic phase transition temperature at which a first-order phase transition occurs. Some phenomenological theories have been propsed to investigate nematicisotropic transition and Maier-Saupe theory (MST) [2] is one of the most commonly used theories. MST has been firstly generalized within Tsallis thermostatistics (TT) in [3] recently. In this study, we aims to investigate the free energy vs the order parameter and specific heat vs temperature by using generalized MST.

According to the MST, the orientational energy of a single molecule in a field generated by its neighbours is

$$
u_{i}\left(\theta_{i}\right)=-\frac{A}{V^{2}} \bar{P}_{2}\left(\frac{3 \cos ^{2} \theta_{i}-1}{2}\right)
$$


where $A$ depends on molexular property and $V$ is the molar volume. One can use this potential function to calculate the orientational contribution to all thermodynamic quantities. The Helmholtz free energy can be calculated by

$$
F=U-T S
$$

where $S$ is the entropy of the system and $U$ is the internal energy of the system. It should be noted that according to MST, the free energy function is found to vanish when the temperature is $T=A / 4.541, k V^{2}$ and the first-order phase transition takes place. Additionally, the order parameter is predicted to be a universal value at the transition point, namely $\left(\bar{P}_{2}\right)_{c}=0.429$, where $c$ index denotes the critical value at the critical temperature. However this prediction is not qualitative aggrement with experiment [4]. Similar discrepancies between the theory and experiment have also been demonstrated by electron resonance studies of a variety of nematogens[5]. The use of molecular field theory might have caused the discrepancy between MST and experiment. Considering this prediction, some authors tried to improve MST by adding higher rank interactions to potential function (2) [6,7] and they improved MST to a higher accuracy of degree. Since it is well known that TT is useful to study the systems having long-range interactions, we use it to see if the above discrepancy removes.

It is well-known that (TT) is successful in the systems $i$ ) have long-range interactions, ii) have long-range memory effects, iii) evolve in multi-fractal space-time.

The orientational distribution function for cylindrically symmetric molecules can be expanded formally in a basis of Legendre polynomials,

$$
f(\beta)=\sum_{L=0}^{N}\left(\frac{2 l+1}{2}\right) \bar{P}_{L} P_{L}(\cos \beta)
$$

where $\bar{P}_{L}$ is the order parameter and defined as

$$
\bar{P}_{L}=\int P_{L}(\cos \beta) f(\beta) \sin \beta d \beta
$$

On the other hand, TT [8,9] has been succesfully applied to various areas $[10,11]$. TT formalism is based on the definition of generalized entropy [8]:

$$
S_{q}=-k \frac{1-\sum_{i} p_{i}^{q}}{1-q}
$$

where $q$ is called "entropic index" and is a measure of the nonextensivity of the system, $p_{i}$ 's are probabilities satisfying $\sum_{i} p_{i}=1$ and $k$ is a positive constant. Eq.(6) reduces to well known Boltzmann-Gibbs entropy when $q \rightarrow 1$. TT has three different alternatives for the internal energy constraint. For simplicity, we use the second choice. If needed, it is possible to pass through the third choice by substituting 


$$
\beta^{\prime}=\frac{\beta}{(1-q) \beta U_{q}+\sum_{j=1}^{\Omega} p_{j}^{q}}
$$

The second choice for the internal energy constraint is given by

$$
U_{q}=\sum_{i=1}^{\Omega} p_{i}^{q} \mathcal{E}_{i}
$$

where $i$ is a given state with energy $\varepsilon_{i}, \Omega$ denotes possible states. If maximizing the generalized entropy given by Eq.(6) according to the constraint (8), one obtains the following probabilities:

$$
p_{i, q}=Z_{q}^{-1}\left[1-(1-q) \beta\left(\varepsilon_{i}-U_{q}\right)\right]^{1 / 1-q}
$$

with

$$
Z_{q}(\beta)=\sum_{i=1}^{\Omega}\left[1-(1-q) \beta\left(\varepsilon_{i}-U_{q}\right)\right]^{1 / 1-q}
$$

The $q$-expectation value of any observable $O$ is then defined as

$$
\langle O\rangle_{q}=\sum_{i=1}^{\Omega} p_{i}^{q} O_{i}
$$

It is here important to solve Eq.(11) which is a self-consistent equation and can be solved numerically.

If we use the equations above, then the second rank order parameter can be calculated using

$$
\left(\bar{P}_{2}\right)_{q}=\left(Z_{q}\right)^{-1} \int_{0}^{\pi / 2} P_{2}(\cos \beta) \exp _{q}\left(a_{2} P_{2}(\cos \beta)\right) \sin \beta d \beta, L \text { even }
$$

where $a_{2}=\bar{u}_{2} \bar{P}_{2} \beta, \bar{u}_{2}=4.541 / \beta_{N I}$ denotes the nematic-isotropic transition temperature and

$$
Z_{q}=\int_{0}^{\pi / 2} \exp _{q}\left(a_{2} P_{2}(\cos \beta)\right) \sin \beta d \beta, L \text { even } .
$$

The corresponding free energy and specific heat of the nematic phase are calculated from

$$
F_{q}=U_{q}-T S_{q}
$$


and

$$
\left(C_{v}\right)_{q}=T\left(\frac{\partial S_{q}}{\partial T}\right)
$$

respectively. The condition for equilibrium is as follows:

$$
\left(\frac{\partial F_{q}}{\partial \bar{P}_{2}}\right)_{V, T}=0 .
$$

This equaiton is called consistency relation or equilibrium condition. The minimization of the free energy occurs at that value of $\bar{P}_{L}$ which satisfies the consistencey relation.

\section{RESULTS AND DISCUSSION}

Figure 1 shows the variation of the free energy vs the order parameter in $P A A(p a r a-a z o x y a n i s o l e)$ at the nematic-isotropic transition temperature. The $q=1$ case denotes the standard MST curve. It is well known from the literature that MST gives a universal value for $\left(\bar{P}_{2}\right)_{N I}=0.429$ [4]. However experimentally, $\left(\bar{P}_{2}\right)_{N I}$ varies in the range of 0.25-0.5 for different compounds [12]. Figure 1 shows that generalized MST within TT predicts different values about the order parameter at the transition temperature and this conclusion could imply that generalized MST within TT could be a suitable tool to investigate the nematic-isotropic transition. The minima for the free energy with respect to the order parameter occur at $\left(\bar{P}_{2}\right)_{N I} \cong 0.39,0.41,0.43,0.45$ and 0.47 for $q=0.98,0.99,1,1.01$ and 1.02 respectively. It seems that with a small departure from the standard MST, one could be able to explain some of the experimental data in the nematic-isotropic transition. 


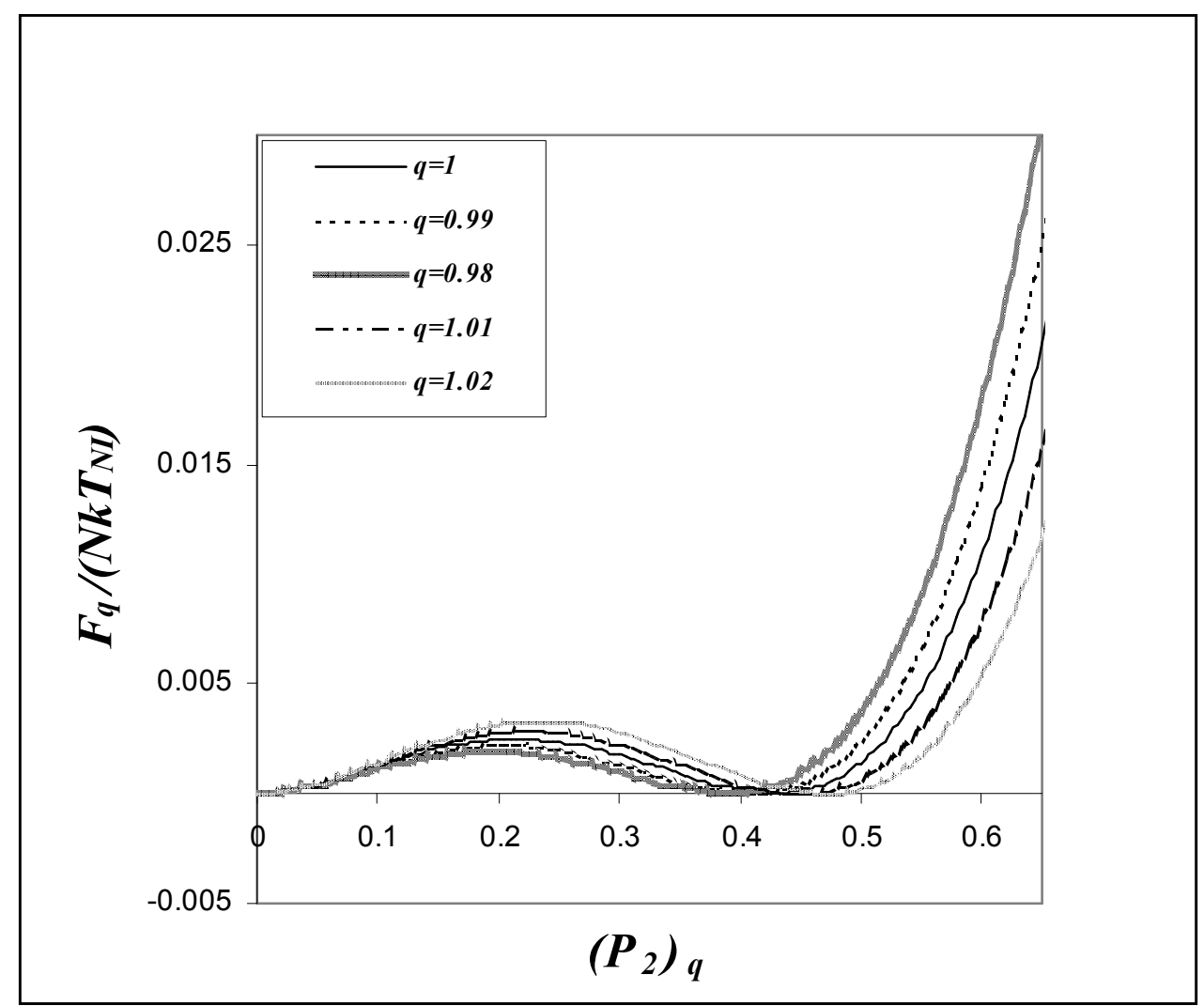

Figure 1. The free energy vs $\bar{P}_{2}$ for PAA at $T_{N I}$ for various values of the entropic index $q$.

The variation of the specific heat vs temperature for $P A A$ is illustrated in Figure 2, where $T / T_{N I}$ is the reduced temperature ( $T_{N I}$ is the nematic-isotropic temperature). As $q$ decreases, the behaviour at the critical temperature becomes more clear. The effect of the nonextensivity is shown in both of figures. If $q \rightarrow 1$, then the results obtained above are reduced to MST ones. 


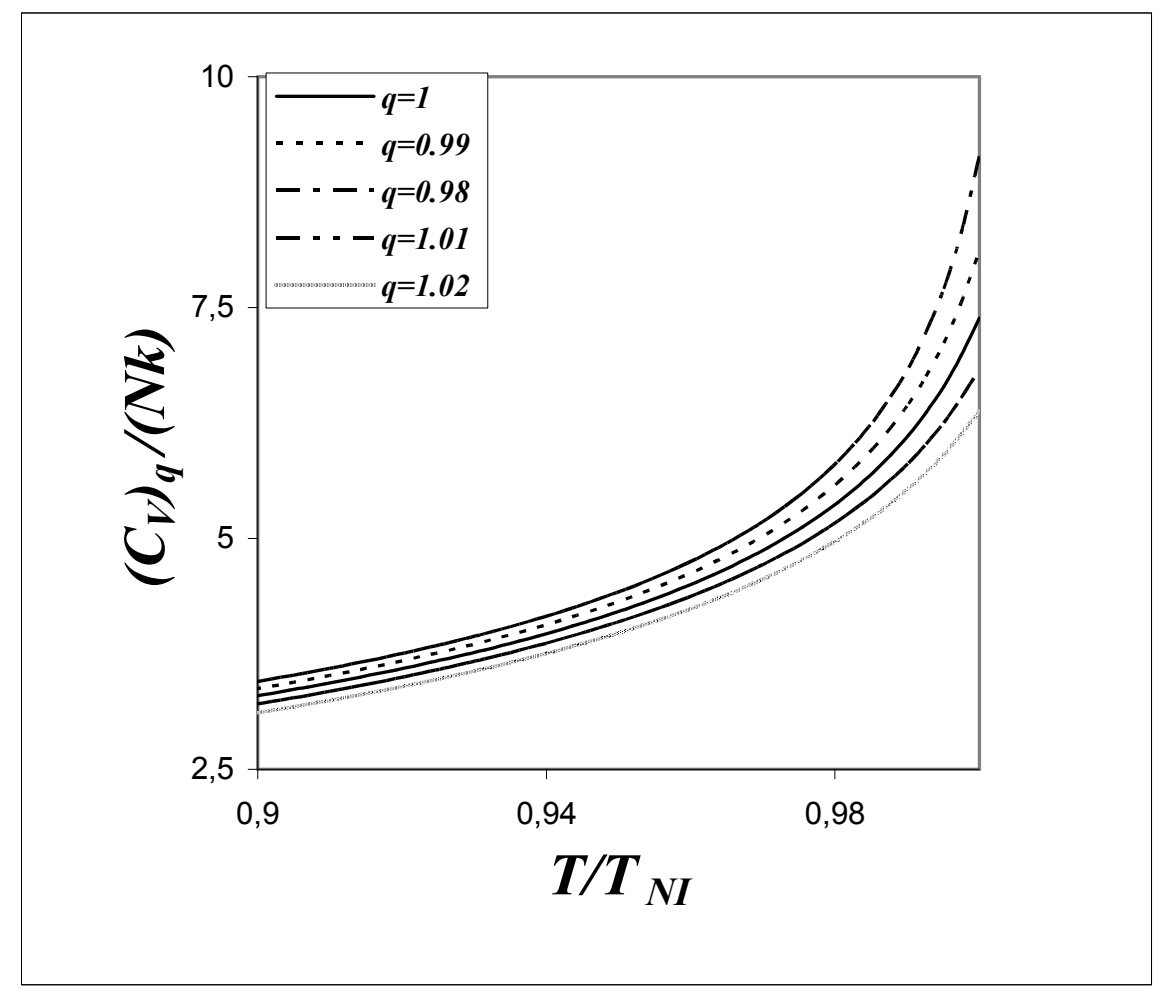

Figure 2. The specific heat vs temperature for PAA for some $q$ values

\section{REFERENCES}

1. S. Chandrasekhar, Liquid Crystals, Cambridge, New York, 1977.

2. W. Maier, A. Saupe, A simple molecular statistical theory of the nematic crystalline-liquid phase II, Z. Naturforsch,. 15a, 287, 1960.

3. O. Kayacan, F. Büyükkılıç, D. Demirhan, Generalization of the Maier-Saupe theory of the nematics within Tsallis thermostatistics, Physica A 301, 255, 2001.

4. S. Singh, Phase Transitions in liquid crystals, Physics Reports, 324, 107, 2000.

5. D.H. Chen, P.G. James, G.R. Luckhurst, Mol. Cryst. Liq. Cryst. 8, 71, 1969.

6. R.L. Humphries, P.G. James, G.R. Luckhurst, Molecular field treatment of nematic liquid crystals, J. Chem. Soc. Faraday Trans. II 68, 1031, 1972.

7. S. Chandrasekhar, N.V. Madhusudana, Statistical theory of orientational order in nematic liquid crystals, Mol. Cryst. Liq. Cryst. 10, 151, 1970.

8. C. Tsallis, Possible generalization of Boltzmann-Gibbs statistics, J. Stat. Phys. 52, 479, 1988.

9. C. Tsallis, R.S. Mendes, A.R. Plastino, The role of constraints within generalized nonextensive statistics, Physica A 261, 534, 1998.

10. S. Abe, Y. Okamoto, Nonextensive statistical mechanics and its applications, Series Lectures Notes in Physics, Berlin: Springer-Verlag, 2001.

11. see http: \tsallis.cat.cbpf.br for an updated bibliography.

12. A. Beguin, J.C. Dubois, P. Le Barny, J. Billard, F. Bonamy, J.M. Busisine, P. Cuvelier, Sources of thermodynamic data on mesogens, Mol. Cryst. Liq. Cryst. 115, $1,1984$. 